\title{
Analysis of Data from Experiments Using Double Labeling
}

\author{
John A. Jacquez, ${ }^{*}+$ David M. Foster, ${ }^{*}$ and Michael F. Shanahan* \\ *Department of Physiology, The Medical School and + Department of Biostatistics, \\ The School of Public Health, The University of Michigan Ann Arbor, Michigan 48109
}

Received July 12, 1976; accepted September 9, 1977

\begin{abstract}
Frequently as a result of experiments in which two isotopes are used one is left with a sequence of samples, the ratio of labeling in each sample, and the problem of analyzing the ratios. Suppose that the experiments are designed so that one expects uniform labeling except for one or two special groups of samples. The problem, then, is to find these groups. Because of the variability in the count rate from sample to sample, the variance of the ratios differs from sample to sample making statistical analysis difficult. Furthermore, there is significant serial correlation in the sample disintegrations per minute for each of the isotopes. We have found that the serial correlation in the labeling ratio is small and of questionable significance in controls but becomes significant when there is a subsequence of samples in which the labeling ratio differs from that in the remainder of the gel. We examine the analysis of variance as a test for significant deviations in the labeling ratio and suggest a method for plotting deviations of labeling ratio from the average background labeling ratio. Finally, we develop a method of estimating the mean labeling ratio from the regression of disintegrations per minute of one isotope on those of the other isotope. This provides another way of plotting deviations in labeling ratio in terms of the residuals around the line of regression.
\end{abstract}

It is often the case in experimental designs which utilize double labeling that one step in the protocol results in a sequence of samples. The ratio of labeling in each sample can be determined, and the problem becomes one of gaining information about the process under consideration from the sequence of ratios thus obtained.

In this paper we examine the problem of analyzing a sequence of ratios when the experiments are designed so that we can assume a constant labeling ratio. The idea behind this type of design, indeed the whole point in using double labeling in this situation, is that the experiments are designed so that the labeling ratio is constant except for one or perhaps a few special subsequences of the sample. The problem is to devise a simple, uniform method for detecting these special subsequences.

We first encountered this problem in attempts to label proteins of the red cell membrane that might be involved in sugar transport. It is known that fluorodinitrobenzene (FDNB) reacts irreversibly with a number of groups on proteins. In the course of its reaction with cell membrane pro- 
teins, glucose transport is inhibited. Cytochalasin B (CB), an inhibitor of glucose transport among other things, slows down the inactivation of glucose transport by FDNB $(1,2)$. We devised a protocol in which a suspension of washed human red blood cells was split and one half was reacted with $\left[{ }^{3} \mathrm{H}\right] \mathrm{FDNB}$ in the presence of $\mathrm{CB}$ while the other half was reacted with $\left[{ }^{14} \mathrm{C}\right] \mathrm{FDNB}$ with no $\mathrm{CB}$ present. The reaction conditions were identical except that the specific activities for the two labels were not the same. After the reaction was terminated the two batches were mixed and their membranes were isolated and solubilized with sodium dodecyl sulfate (SDS) and were then electrophoresed on polyacrylamide gels (PAGESDS) (3). The gels were sliced into 1- to 2-mm slices thus generating a sequence of samples, and each slice was counted with use of a doublelabel counting technique in a scintillation counter. Although the total labeling differs in different slices, we expect that the ratio of ${ }^{14} \mathrm{C}$ to ${ }^{3} \mathrm{H}$ label will be constant except where there are proteins whose rate of reaction with FDNB was changed by the cytochalasin B. Protocols that use variations on this same general idea have been used widely (4-7).

Although we will examine the analysis of data generated in the experiments described above, the analysis holds for a variety of experiments that use the same idea. Moreover, ours is not the first effort to develop a suitable theory; there have been previous attempts to analyze data from such experiments that involve labeling with two isotopes (4-7). In fact Weisberg's analysis (5) was the starting point for our work. However, none of these previous attempts (4-7) takes into account the fact that not only are the estimated disintegrations per minute for the two isotopes in a slice correlated, but there is also a serial correlation between nearby slices. Our initial attempts suffered from the same deficiencies.

In what follows we first discuss the two major difficulties encountered in the analysis and interpretation of the experiments and then examine two approaches to the problem.

\section{THE PROBLEM AND ITS DIFFICULTIES}

In Fig. 1 we show the standard way of plotting data on the ratio of the two labels. These data come from one of our control runs in which there was no difference in treatment of the two suspensions, so the ratio of labeling should have been constant. In the upper curve are plotted the total disintegrations per minute (DPM) in tritium to show how much variation there is in the labeling. The lower curve shows the ratio of DPM, ${ }^{14} \mathrm{C} /{ }^{3} \mathrm{H}$. There appears to be a greater variability in the ratio in the first few slices which are the ones with the lowest counts. Figure 2 shows another such plot but for a preparation which shows differential labeling resulting from the effect of $\mathrm{CB}$ on the FDNB reaction. The subsequence of slices $31-41$ shows a large change in the labeling ratio, and there is a suggestion 

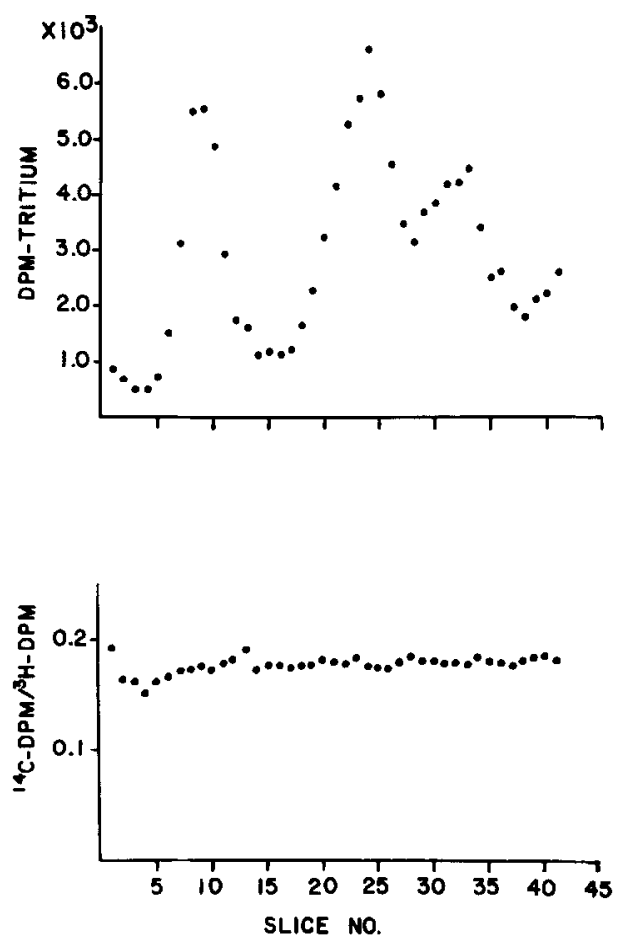

FiG. 1. Disintegrations per minute (DPM) in tritium and ${ }^{14} \mathrm{C} / 3 \mathrm{H}$ labeling ratio in a control run. Two groups of human red blood cells were reacted with FDNB, one with $\left[{ }^{14} \mathrm{C}\right]$ FDNB, the other with $\left[{ }^{3} \mathrm{H}\right] \mathrm{FDNB}$, under identical conditions except that DMSO, which was used in our cytochalasin B solutions, was present in one of the reactions. The two groups were washed, combined, and hemolyzed, and a suspension of the ghosts was prepared. The ghosts were dissolved in SDS and run on SDS-PAGE.

of a deviation in slices 20-27. Usually the experimenter compares the experimental result with that in a control run to decide whether the deviation in a subsequence is significant, although statistical tests of significance are usually not used. In the experiment shown in Fig. 2, the changes in labeling ratio in slices $31-41$ are unusually prominent.

The first difficulty in evaluating the significance of any deviations from the mean ratio is that the variance of the ratio may vary considerably, being high in regions of low total counts and much lower in slices that have high count rates. Judgment as to the relative importance of deviations in labeling ratio would be aided by transforming to a variate that has constant or even nearly constant variance. Furthermore, the variance of the ratio of two random variables reduces to an easily usable expression only if the standard deviation of the denominator is small in relation to its mean value.

The other difficulty is that of serial correlation in the sequence of DPM 

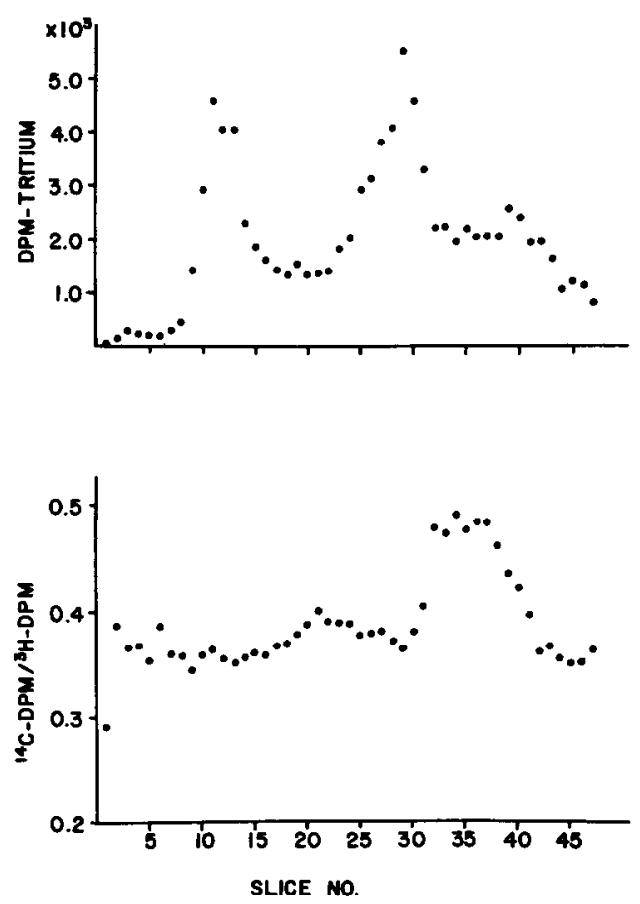

Fig. 2. DPM in tritium and ${ }^{14} \mathrm{C} /{ }^{3} \mathrm{H}$ labeling ratio. In this case $80 \mu \mathrm{M}$ cytochalasin $\mathrm{B}$ (CB) was present in the reaction mixture of the red blood cells (rbc) which were reacted with $\left[{ }^{3} \mathrm{H}\right] \mathrm{FDNB}$, and 2-deoxyglucose was present during the reaction of the rbc which were reacted with $\left[{ }^{14} \mathrm{C}\right] \mathrm{FDNB}$. Later it was shown that 2-deoxyglucose has no effect at the concentrations used and that the same results could be obtained with $\mathrm{CB}$ alone.

in ${ }^{14} \mathrm{C}$ and in ${ }^{3} \mathrm{H}$. Figure 3 is a plot of the circular serial correlation (8) for the DPM in tritium in the control run shown in Fig. 1. The serial correlations for lags of one and two slices, $R_{1}$ and $R_{2}$, are usually significant, and one has to go to $R_{3}$ or $R_{4}$ and occasionally $R_{5}$ before the autocorrelation becomes insignificant for controls. It is possible to run the experiments so that the correlation is negligible between adjacent slices, by putting very little protein on the gels. Then the proteins appear as sharp bands, almost like a line spectrum, and if the slices are made considerably thicker than the bands, the correlation between adjacent slices can be practically eliminated. The difficulty is that when this is done the total number of counts per slice decreases markedly, and so the counting error is greatly increased. In order to obtain high count rates the gels are usually "overloaded." In overloaded gels the bands spread out, and adjacent bands may overlap more or less depending on their separation. This is the reason for the serial correlation shown in Fig. 3. It is possible at times, though more difficult technically, to use large amounts of pro- 


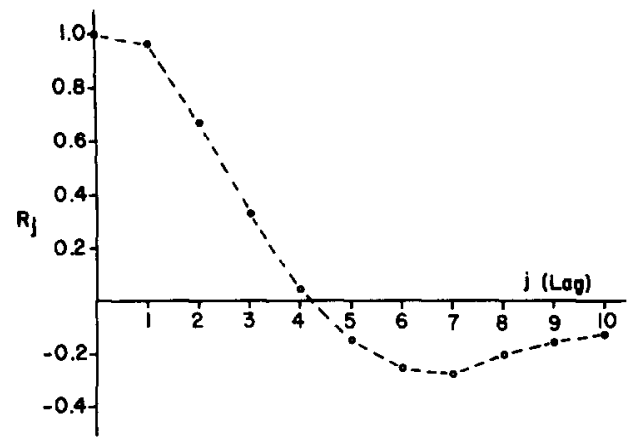

Frg. 3. Circular serial correlation coefficient, $R_{j}$, for the $\mathrm{H}^{3} \mathrm{DPM}$ in the gel slices of the control run shown in Fig. 1 for lags of 0 to 10. The first 41 slices werc used because theory requires that the number of sequential samples be a prime number [see ref. (8)].

teins and so obtain high count rates and still have narrow bands by using gel tubes of larger diameter than the standard tubes.

Figure 4 shows the serial correlation for the ${ }^{3} \mathrm{H}$ DPM for the experimental run shown in Fig. 2, for the first 31 slices and for the first 47 slices. Again the serial correlation is significant. Fortunately the serial correlation decreases markedly in the ratio. Figure 5 shows the serial correlation for the ratio ${ }^{14} \mathrm{C} /{ }^{3} \mathrm{H}$ DPM for the control run in the upper panel and for the experimental run in the lower panel. Knowing that there are sometimes problems with stability of the variance of a ratio, one must be more circumspect in interpreting Fig. 5. On the face of it, the serial correlation is negligible for the first 31 slices but becomes significant when slices $32-41$, those with the marked deviation in ratio, are included. We have designed our experiments so as to obtain high DPM due to tritium (the

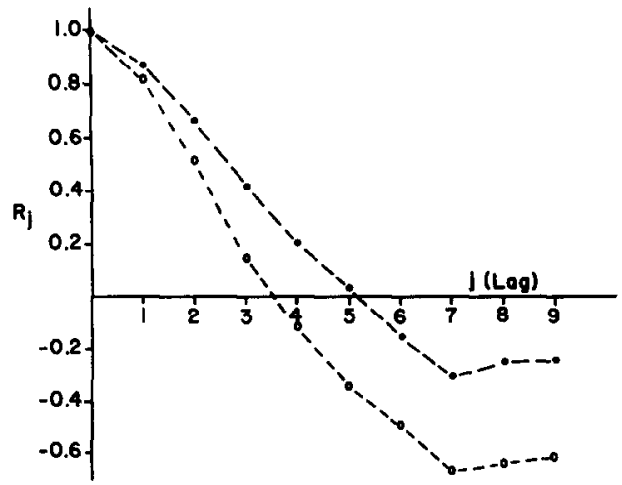

FIg. 4. The circular serial correlation coefficient for the $\mathrm{H}^{3} \mathrm{DPM}$ in the gel slices of the CB experiment shown in Fig. 2. The correlation coefficient was calculated using the first 31 slices (-O-) and the first 47 slices (-O). 
denominator in the ratio) in order to improve stability of the variance of the ratio and are inclined to accept Fig. 5 at face value.

\section{ESTIMATES OF BASIC VARIANCES}

Since we need estimates of the variances of the calculated disintegrations per minute (DPM) of the two isotopes, we will begin by developing the theory for tritium $\left({ }^{3} \mathrm{H}\right)$ and carbon-14 $\left({ }^{14} \mathrm{C}\right)$ where two channels are used for counting the doubly labeled samples. For other isotopes, the formulas for the standard deviations of the DPM may require slight modifications.

In this method of counting, the carbon channel is set so that only ${ }^{14} \mathrm{C}$ is counted, the spillover from tritium being negligible. In the tritium channel, both tritium and ${ }^{14} \mathrm{C}$ are counted, but with different efficiencies. A discussion of the optimization of counting conditions to maximize precision of counting doubly labeled samples can be found in Bush's paper (9).

We will adopt the following notation:

$C_{C}$ Total counts obtained in the carbon channel

$t_{\mathrm{C}}$ Total count time for the sample in the carbon channel
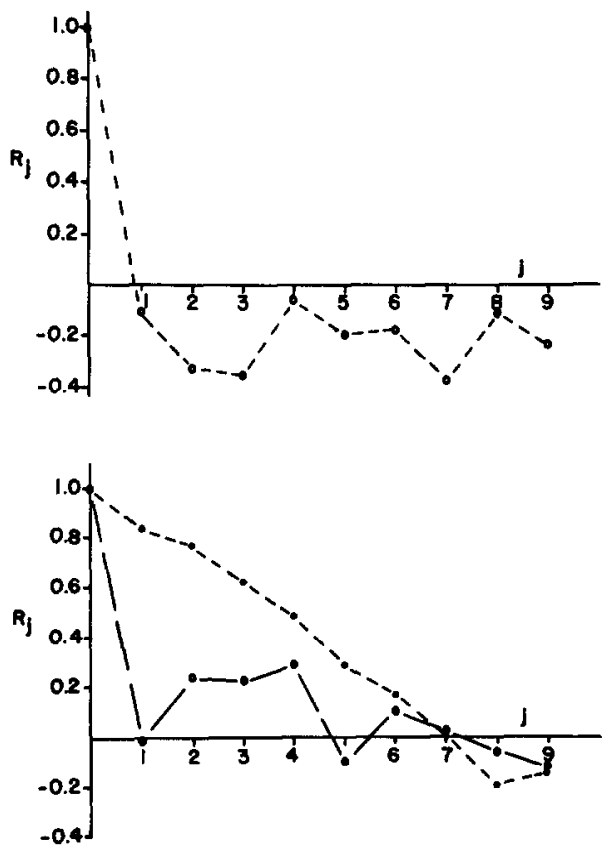

FIG. 5. The circular serial correlation coefficients for the ${ }^{14} \mathrm{C} D P M /{ }^{3} \mathrm{H} D P M$ ratio for the control run of Fig. 1 (upper graph) and for the CB experiment shown in Fig. 2 (lower graph) for the first 31 slices (-O-) and the first 47 slices (-O-). 
$B_{\mathrm{C}}$ Total background count in the carbon channel

$t_{\mathrm{CB}}$ Total time of counting background in the carbon channel

$C_{\mathrm{T}}$ Total counts obtained in the tritium channel

$t_{\mathrm{T}}$ Total count time for the sample in the tritium channel

$B_{\mathrm{T}}$ Total background count in the tritium channel

$t_{\mathrm{TB}}$ Total time of counting background in the tritium channel

$f_{\mathrm{CC}}$ Efficiency of counting ${ }^{14} \mathrm{C}$ in the carbon channel

$f_{\text {TC }}$ Efficiency of counting ${ }^{14} \mathrm{C}$ in the tritium channel

$f_{\text {Tт }}$ Efficiency of counting ${ }^{3} \mathrm{H}$ in the tritium channel

The background counts in the two channels are distributed independently of the sample counts in the channels. We may assume that the rates of disintegration of ${ }^{14} \mathrm{C}$ and ${ }^{3} \mathrm{H}$ are independent Poisson-distributed variables. The estimate of the disintegrations per minute due to ${ }^{14} \mathrm{C}$ $\left(\mathrm{DPM}_{\mathrm{C}}\right)$ is calculated from the counts in the carbon channel using Eq. [1].

$$
\mathrm{DPM}_{\mathrm{C}}=\frac{1}{f_{\mathrm{CC}}}\left(\frac{C_{\mathrm{C}}}{t_{\mathrm{C}}}-\frac{B_{\mathrm{C}}}{t_{\mathrm{CB}}}\right)
$$

The total counts per minute in the tritium channel corrected for background $\left(\mathrm{CPM}_{\mathrm{T}}\right)$ is given by Eq. [2].

$$
\mathrm{CPM}_{\mathrm{T}}=\frac{C_{\mathrm{T}}}{t_{\mathrm{T}}}-\frac{\boldsymbol{B}_{\mathrm{T}}}{t_{\mathrm{TB}}}
$$

$\mathrm{CPM}_{\mathrm{T}}$ is related to the DPM due to tritium $\left(\mathrm{DPM}_{\mathrm{T}}\right)$ and $\mathrm{DPM}_{\mathrm{C}}$ by Eq. [3]. Solving this equation for $\mathrm{DPM}_{\mathrm{T}}$ gives Eq. [4].

$$
\begin{aligned}
& \mathrm{CPM}_{\mathrm{T}}=f_{\mathrm{TT}} \mathrm{DPM}_{\mathrm{T}}+f_{\mathrm{TC}} \mathrm{DPM}_{\mathrm{C}} \\
& \mathrm{DPM}_{\mathrm{T}}=\frac{\mathrm{CPM}_{\mathrm{T}}-f_{\mathrm{TC}} \mathrm{DPM}_{\mathrm{C}}}{f_{\mathrm{TT}}}
\end{aligned}
$$

Since $\mathrm{DPM}_{\mathrm{T}}$ is a linear combination of the counts in the two channels, $\mathrm{DPM}_{\mathrm{C}}$ and $\mathrm{DPM}_{\mathrm{T}}$ are correlated. The sample estimates of the variances and covariances are given by Eqs. [5]-[7].

$$
\begin{aligned}
& s_{\mathrm{C}}^{2}=\frac{1}{f_{\mathrm{CC}}{ }^{2}}\left(\frac{C_{\mathrm{C}}}{t_{\mathrm{C}}{ }^{2}}+\frac{B_{\mathrm{C}}}{t_{\mathrm{CB}}{ }^{2}}\right) \\
& s_{\mathrm{T}}{ }^{2}=\frac{1}{f_{\mathrm{TT}^{\prime}}{ }^{2}}\left[\frac{C_{\mathrm{T}}}{t_{\mathrm{T}}{ }^{2}}+\frac{B_{\mathrm{T}}}{t_{\mathrm{TB}}{ }^{2}}+\frac{f_{\mathrm{TC}}{ }^{2}}{f_{\mathrm{CC}^{2}}{ }^{2}}\left(\frac{C_{\mathrm{C}}}{t_{\mathrm{C}}{ }^{2}}+\frac{B_{\mathrm{C}}}{t_{\mathrm{CB}}{ }^{2}}\right)\right]
\end{aligned}
$$

$\operatorname{cov}\left(\mathrm{DPM}_{\mathrm{C}}, \mathrm{DPM}_{\mathrm{T}}\right)=-\frac{f_{\mathrm{TC}}}{f_{\mathrm{TT}} f_{\mathrm{CC}}{ }^{2}}\left(\frac{C_{\mathrm{C}}}{t_{\mathrm{C}}{ }^{2}}+\frac{B_{\mathrm{C}}}{t_{\mathrm{CB}}{ }^{2}}\right)=-\frac{f_{\mathrm{TC}}}{f_{\mathrm{TT}}} s_{\mathrm{C}}{ }^{2}$

In the above equations we have treated the efficiencies and the counting time as constants. They are of course subject to some error but can be 
obtained to relatively high accuracy in comparison with the counts in the two channels.

Our experiments are usually designed so that there are more counts from ${ }^{3} \mathrm{H}$ than from ${ }^{14} \mathrm{C}$. Then, because the spillover of ${ }^{14} \mathrm{C}$ into the tritium channel is small, the term due to ${ }^{14} \mathrm{C}$ counts in Eqs. [4] and [6] is small. The covariance is therefore small in relation to $s_{\mathrm{T}}{ }^{2}$ but it is still an appreciable fraction, $0.2-0.5$, of $s_{\mathrm{C}}{ }^{2}$, and if quenching is high the covariance can become 0.8 or more of $s_{\mathrm{C}}{ }^{2}$ in absolute value.

In what follows we use the notation $x_{i}$ for the estimated DPM in slice $i$ for the isotope that has the highest overall DPM, and $y_{i}$ for the other. In all of our applications $x$ refers to the DPM due to tritium and $x_{i}>y_{i}$ for all $i$.

\section{THE LABELING RATIO FOR GELS}

\section{(a) The Labeling Ratio and its Variance}

One intuitively obvious way to approach the problem is to look at the ratios $y_{i} / x_{i}$. In fact in the majority of publications on such studies the data are reported as labeling ratios without any considerations of statistical significance. Unfortunately, we do not have exact simple expressions for the variance of the ratio of two correlated random variates. However, if the denominator has a sharp distribution, i.e., $\mu_{x_{i}}$, large in comparison with $\sigma_{x_{i}}$ then a good approximation for the variances of the ratios can be obtained. We obtain this by making sure we have high counts in the denominator and by counting the slices long enough so that $s_{x} / x$ is less than 0.01 for most gel slices.

Consider two random variates, $X$ and $Y$. Let $Z=Y / X$. Then one can show that to second-order terms the mean, $\mu_{Z}$, is given by Eq. [8]. The variance, to third-order terms, is given by Eq. [9].

$$
\begin{aligned}
& \mu_{Z}= \frac{\mu_{Y}}{\mu_{X}}-\frac{\mu_{X Y}}{\mu_{X}^{2}}+\frac{\mu_{Y}}{\mu_{X}^{3}} \sigma_{X}^{2} \\
& \sigma_{Z}^{2}=\left(\frac{\sigma_{Y}^{2}}{\mu_{X}^{2}}-\frac{2 \mu_{Y}}{\mu_{X}^{3}} \mu_{X Y}+\frac{\mu_{Y}^{2}}{\mu_{X}^{4}} \sigma_{X}^{2}\right) \\
& \quad-2\left(\frac{\mu_{Y^{2} X}}{\mu_{X}^{3}}-\frac{2 \mu_{Y}}{\mu_{X}^{4}} \mu_{X Y^{2}}+\frac{\mu_{Y}^{2}}{\mu_{X}^{5}} \mu_{X^{3}}\right)
\end{aligned}
$$

In these equations $\mu_{X m Y n}$ are the $m+n$ order central moments except that as usual $\sigma_{Y}^{2}$ and $\sigma_{X}^{2}$ are used for $\mu_{y^{2}}$ and $\mu_{X^{2}}$, respectively.

Let $z_{i}=y_{i} / x_{i}$ where $x_{i}$ is the DPM for the isotope which, overall, has the smallest ratio of standard deviation to mean value, $s_{x} / x$. Then if the DPM in the $x_{i}$ are large enough so that the $x_{i}$ can be considered to 
have a very peaked distribution, we use the sample estimates $s_{z_{i}}^{2}$ for $\sigma_{z_{i}}^{2}$ and in so doing retain only the second-order terms from Eq. [9].

$$
s_{z_{i}}^{2}=\frac{s_{y_{i}}^{2}}{x_{i}{ }^{2}}-\frac{2_{y_{i}}}{x_{i}{ }^{3}} \operatorname{cov}\left(x_{i}, y_{i}\right)+\frac{y_{i}{ }^{2}}{x_{i}{ }^{4}} s_{x_{i}}^{2}
$$

\section{(b) Statistical Testing}

The derivation of rigorous tests of statistical significance of deviations is difficult. It is well known that there are difficulties in obtaining confidence limits for the ratio of two normally distributed random variates (10). On the other hand confidence limits are readily obtained for the ratio of two Poisson-distributed random variates, $x_{1} / x_{2}$, conditional on $x_{1}+x_{2}=n$ being fixed $(11,12)$. Our DPM are derived from Poissondistributed random variates, the direct counts in the two channels of the counter. More to the point, our experience with our control gels is that, provided we have high count rates and provided that we count our samples long enough so that $s_{x} / x$ is quite small, the ratios from control gels are quite stable. However, rigorous tests are not really needed; the judgment of the experienced investigator plays the major role in decisions of experimental significance of deviations. For example, the experienced investigator is loath to accept a single point with a deviant ratio as significant even if a rigorous statistical test were significant because he would assume that some accident in handling or counting that slice could have intervened. One would be reluctant to come to such a conclusion even with two successive points that deviate markedly from the mean. However, a sequence of points deviating to one side of the mean, occurring in the same location on the gels in replicate studies, is convincing. When such a deviation is not found in control gels and when one can demonstrate a relation between magnitude of the deviation and magnitude of an experimental control variable, one has evidence for a significant effect. For example Fig. 6 shows that the magnitude of the deviation in the ratio in our studies increases with the concentration of CB. This, with the demonstration that the effect does not occur in appropriate controls, is the evidence that convinces the investigator of the significance of the effect.

Our point is that the evidence for the significance of an effect comes from the overall experimental design, and that statistical tests on the sequence of gel slices from any one run play a secondary role. Nonetheless, it would help the investigator to have simple approximate tests to help him.

\section{(c) Analysis of Variances}

Since the serial correlation for the ratios in the controls is not significant, a rough test would be to divide the gel slices into two groups, those 

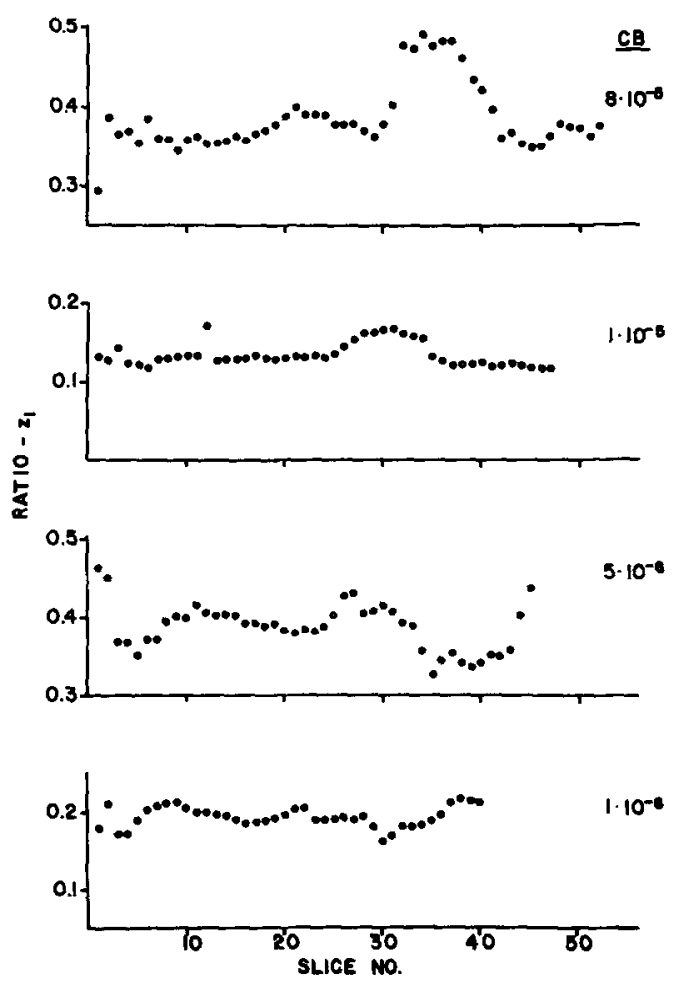

Fig. 6. The ${ }^{14} \mathrm{C} \mathrm{DPM} /{ }^{3} \mathrm{H}$ DPM ratios from four experiments with different concentrations of CB present. The gels were not all of the same length; the deviation in slices $31-41$ for the gel from the $8 \cdot 10^{-5} \mathrm{M} C B$ experiment and that in slices $26-34$ for the $1 \cdot 10^{-5} \mathrm{M} \mathrm{CB}$ experiment occur at the same place in the electrophoretogram. There is little effect on the labeling ratio at $10^{-6} \mathrm{M} \mathrm{CB}$, but there is a significant effect at $5 \cdot 10^{-6} \mathrm{M}$. For the experiment at $5 \cdot 10^{-6} \mathrm{M} C B$, the experiment was run so that a decrease in ratio is expected if $C B$ affects the labeling ratio; there is a decrease in the region where it is expected. slices 34-43.

that deviate considerably from the mean ratio and the remainder, leaving out the few slices that show intermediate ratios. The idea is to try to divide the slices into two groups neither of which shows significant serial correlation. For example, for the experiment shown in Fig. 2, slices 3238 could form one group, slices $1-30$ plus $41-47$ the other group. A simple rough test would be to use the one-way analysis of variance assuming equal variance for the ratios of the slices in each group. The $F$ test using the ratio of the mean squares between means to the within-group mean square would provide the test. Since the problem does not meet all the assumptions for the $F$ test, the test is approximate but one can compensate for its approximate nature by using fewer degrees of freedom than calculated for the two groups. 
The simplest statistical model for the ratio of the $i$ th slice can be assumed to be $z_{i}=\mu+\alpha_{i}+\epsilon_{i}$ where $\mu$ is the mean ratio and $\alpha_{i}$ comes from a distribution of mean zero and common variance $\sigma^{2} ; \alpha_{i}$ represents the variation in labeling ratio due to variation in the labeling process. Having drawn a sample of ratio $\mu+\alpha_{i}$, we then measure it with measurement error $\epsilon_{i}$ which comes from a distribution of mean zero and variance $\sigma_{i}{ }^{2}$ that differs from one slice to another. For this model, we base no exact $F$ test on the one-way analysis of variance; however, the above rough test is probably adequate.

\section{(d) Circular Serial Correlation}

Probably the easiest test to use is the standard serial correlation coefficient. We have used the circular serial correlation coefficient because it is easy to use computationally and there are exact confidence limits available. There are no reasons in the structure of the data to use the circular serial correlation coefficient, but for low-order correlation coefficients the values obtained do not differ significantly from the standard serial correlation coefficients. If the controls show no significant serial correlation and the experimental run, truncated to exclude the deviant ratios, shows no significant serial correlation but the full experimental gel does show significant serial correlation, one has evidence for a significant effect. This is what is shown by Fig. 5. Note that the circular serial correlation is not significant in the control run (upper panel) or for the first 31 slices of the experimental run. Inclusion of slices $32-47$ gives a marked serial correlation due to the change in labeling ratio in slices 32-41. The calculation of the serial correlation coefficient for lags $j=1,2, \ldots$, and so on is simple, and the values the coefficient must attain to be significant at the 5 and $10 \%$ levels are tabulated (8).

\section{(e) Plotting of Data}

For analysis of the effect of changing one of the experimental variables it would be most useful to have a way of quantitating the deviation in the region of differential labeling in a way that takes into account the variation in errors of the labeling ratio in the slices. For example, it would be useful to be able to plot the total deviation in labeling ratio against the concentration of cytochalasin B for the data shown in Fig. 6 in terms of some normalized variable which takes into account the different errors in different slices in one run as well as the difference in errors between runs. We have found the following to be useful. If changing an experimental variable appears to give a shift in labeling ratio of the electrophorogram we calculate the weighted mean ratio for the slices from such gels, excluding the slices from the region of differential labeling. Thus we 
calculate $\bar{z}$ by Eq. [11] where it is understood that the index $i$ does not include the slices from the region of differential labeling.

$$
\bar{z}=\frac{\sum_{i} z_{i} / s_{z_{i}}}{\sum_{i} 1 / s_{z_{i}}}
$$

The slices from this region are excluded from the calculation of the weighted mean ratio for all gels including the control gels for the experiment. We then calculate the deviation $\left(z_{k}-\bar{z}\right)$ for each slice $k$ and normalize this by dividing by the estimated standard deviation of $\left(z_{k}-\bar{z}\right), s_{k}$, to give a normalized residual, Eq. [12].

$$
r_{k}=\frac{z_{k}-\bar{z}}{s_{k}}
$$

The variances for $\left(z_{k}-\bar{z}\right)$ are given by Eqs. [13] and [14] in which $n$ is the number of slices used in calculating the mean $\bar{z}$.

$$
\begin{aligned}
& \sigma_{k}^{2}=\sigma_{z_{k}}^{2}-\frac{2 \sigma_{z_{k}}}{\sum 1 / \sigma_{z_{j}}}+\frac{n}{\left(\sum 1 / \sigma_{z_{j}}\right)^{2}} \\
& \sigma_{k}^{2}=\sigma_{z_{k}}^{2}+\frac{n}{\left(\sum 1 / \sigma_{z_{j}}\right)^{2}}
\end{aligned}
$$

Equation [13] holds if slice $k$ is included in the set used to calculate $\bar{z}$; Eq. [14] holds for those slices which come from the region not included in the weighted mean. We have used Eqs. [15] and [16] in which the variances of Eqs. [13] and [14] have been replaced by the sample estimates, $s_{z_{k}}^{2}$.

$$
\begin{aligned}
& s_{k}^{2}=s_{z_{k}}^{2}-\frac{2 s_{z_{k}}}{\sum_{j} 1 / s_{z_{j}}}+\frac{n}{\left(\sum_{j} 1 / s_{z_{j}}\right)^{2}} \\
& s_{k}^{2}=s_{z_{k}}^{2}+\frac{n}{\left(\sum_{j} 1 / s_{z_{j}}\right)^{2}}
\end{aligned}
$$

Equations [15] and [16] give fairly good estimates of the variances $\sigma_{k}^{2}$ if one makes a point of obtaining high counts from the slices so that $s_{z_{i}}^{2}$ are good estimates of $\sigma_{z_{i}}^{2}$.

The use of this transformation gives results that are in accord with our expectations. For example Fig. 7 shows the data of Fig. 6 replotted in terms of the residuals, $r_{k}$; now there is a more obvious increase in the differential effect on labeling as the concentration of CB is increased. The 


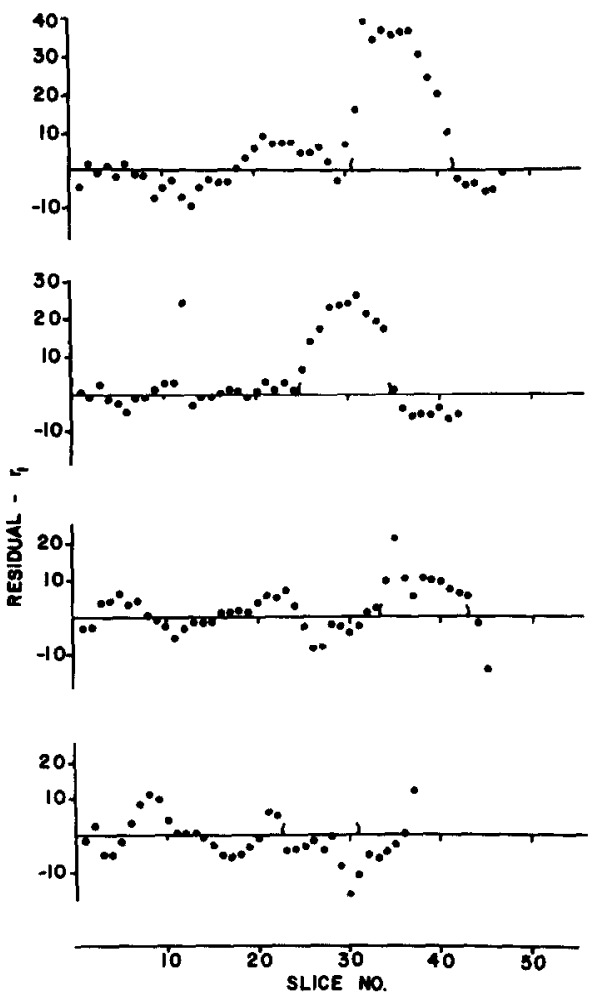

FIG. 7. Plots of the residuals, $r_{i}=\left(z_{i}-\bar{z}\right) / s_{i}$, for the ${ }^{14} \mathrm{C} /{ }^{3} \mathrm{H}$ labeling ratios shown in Fig. 6. For the third curve from the top, $\mathrm{CB}=5 \cdot 10^{-6} \mathrm{M}$; the sign of the residual has been changed so that the expected deviation in the residuals due to the $\mathrm{CB}$ effect would be in the positive direction. The corresponding regions of the electrophoretograms are indicated by the brackets on the abscissas; the slices within these brackets were not included in the calculation of the weighted means, $\bar{z}$, for their respective gels.

region of the electrophoretogram corresponding to the region of differential labeling in the two highest concentrations of $\mathrm{CB}$ is marked on the abscissas of each of the plots in Fig. 7. From plots such as Fig. 7, it is possible to plot the sum of the residuals in the region of differential labeling against the concentration of $\mathrm{CB}$ and thus quantitate the effect of CB. The complete results of this study with a discussion of their implication for the system transporting glucose in erythrocytes will be published elsewhere (13).

\section{THE REGRESSION APPROACH}

An interesting approach is to use the regression of DPM due to one isotope against the DPM due to the other to estimate the mean labeling 
ratio and then to examine the residuals. There are some problems in developing the theory for estimating parameters because of the serial correlation, but these may be partly overcome with the proper iterative model. The basic model is developed below.

Suppose we are given a set of $n$ sequentially numbered samples and suppose $\eta_{i}$ and $\xi_{i}$ are the "true" DPM for the two isotopes, $\xi$ being the one with the higher DPM. Although the extent of labeling might differ for the different samples, our basic assumption is that the relative labeling by the two isotopes is the same for all samples. The null hypothesis, then, is that $\eta_{i}$ and $\xi_{i}$ are in the same ratio $\zeta$ for all samples, from which comes Eq. [17].

$$
\eta_{i}=\zeta \xi_{i}, i=1, \ldots, n
$$

The true values $\eta_{i}$ and $\xi_{i}$ are never observed because the measured DPM contain errors due to random fluctuations in the labeling process and in the counting. Thus the measured values of DPM, $y_{i}$ and $x_{i}$, are related to the true values by Eqs. [18] and [19].

$$
\begin{aligned}
& \eta_{i}=y_{i}+\epsilon_{i} \\
& \xi_{i}=x_{i}+e_{i}
\end{aligned}
$$

Here $\epsilon_{i}$ and $e_{i}$ are random variables with mean zero and variances $\sigma_{y_{i}}^{2}$ and $\sigma_{x_{i}}^{2}$, respectively. Note that for the counting technique which we are using Eqs. [5] and [6], writien for the $i$ th sample, give the sample estimates of these variances.

Now suppose we substitute Eqs. [18] and [19] into Eq. [17]. Doing so gives us Eq. [20].

$$
y_{i}=\zeta x_{i}+\left(\zeta e_{i}-\epsilon_{i}\right)
$$

Thus the model predicts that there is a linear relationship between the measured DPM with a superimposed random fluctuation due to the random variable $\zeta e_{i}-\epsilon_{i}$. This variable has mean zero and variance given by Eq. [21].

$$
\sigma_{i}^{2}=\zeta^{2} \sigma_{x_{i}}^{2}-2 \zeta \operatorname{cov}\left(x_{i}, y_{i}\right)+\sigma_{y_{i}}^{2}
$$

The sample estimate $s_{i}{ }^{2}$ for $\sigma_{i}^{2}$ can be calculated from Eq. [22],

$$
s^{2}=z^{2} s_{x_{i}}^{2}-2 z \operatorname{cov}\left(x_{i}, y_{i}\right)+s_{y_{i}}^{2},
$$

where $z$ is the estimate of $\zeta$ obtained from a least squares fit and $s_{x_{i}}^{2}$, $s_{y_{i}}^{2}$, and $\operatorname{cov}\left(x_{i}, y_{i}\right)$ are given by Eqs. [5], [6], and [7]. The slope $z$ is estimated iteratively with a weighted least squares fit using the weights $w_{i}=1 / s_{i}^{2}$.

If there were no serial correlation it would be simple to find the conditional variance of $z$ given $x_{1}, x_{2}, \ldots, x_{n}$ and to calculate residuals 
around the regression line and to find the sample variance of the residual for each point. Note that $z$ is an unbiased estimator of $\zeta$ whether or not there is serial correlation. However, the presence of serial correlation complicates the estimation of the variances. One of the referees of a prior draft of this paper suggested that the major effects would be captured if we assumed a constant first-order serial correlation and zero higher order correlations. The consequences of this assumption are developed below.

Let $\delta_{i}=\left(\zeta e_{i}-\epsilon_{i}\right)$. Then, we assume Eq. [23].

$$
E\left[\delta_{i} \delta_{j}\right]=\left\{\begin{array}{cl}
\sigma_{i}{ }^{2}=\frac{1}{w_{i}}, i=j \\
\rho \sigma_{i} \sigma_{j}=\frac{\rho}{\left(w_{i} w_{j}\right)^{1 / 2}}, & i=j \pm 1 \\
0 \quad, & |i-j|>1
\end{array}\right.
$$

Now $\rho$ and $\zeta$ are also interrelated and their estimates, $\bar{\rho}$ and $z$, have to be obtained iteratively. To do this one can obtain an initial estimate $z_{0}$ without weighting and then generate $z_{1}$ and $\bar{\rho}_{1}$. Given the estimates $z_{m}$ and $\bar{\rho}_{m}$, $z_{m+1}$ is obtained from the least squares weighted regression using the weights $w_{i}=1 / s_{i}{ }^{2}$ which are calculated with use of $z_{m}$ in Eq. [22], and $\bar{\rho}_{m+1}$ is the serial correlation coefficient of lag 1 for the sequence of $y_{i}-z_{m} x_{i}, i=1, \ldots, n$. With the above assumption it is easy to show that the variance of $z$, conditional on the given values $x_{1}, \ldots, x_{n}$, is given by Eq. [24].

$$
V\left(z / x_{1}, \ldots, x_{n}\right)=\frac{1}{\sum w_{i} x_{i}^{2}}+2 \rho \frac{\sum_{i=1}^{n-1}\left(w_{i} w_{i+1}\right)^{1 / 2} x_{i} x_{i+1}}{\left(\sum w_{i} x_{i}^{2}\right)^{2}}
$$

Now consider the deviation around the fitted line at each point, $y_{k}$, Eq. [25], where $\hat{y}_{k}=z x_{k}$. The variance of $d_{k}$ is given by Eq. [26].

$$
\begin{aligned}
d_{k} & =y_{k}-\hat{y}_{k} \\
v_{k}^{2} & =V\left(y_{k}-\hat{y}_{k} / x_{1}, \ldots, x_{n}\right) \\
& =\sigma_{k}^{2}+x_{k}^{2} V\left(z / x_{1}, \ldots, x_{n}\right)-2 x_{k} V\left[\delta_{k}(z-\zeta) / x_{1}, \ldots, x_{n}\right]
\end{aligned}
$$

Here $\sigma_{k}^{2}$ is given by Eq. [21], $V\left(z / x_{1}, \ldots, x_{n}\right)$ by Eq. [24], and $V\left(\delta_{k}(z-\zeta) / x_{1}, \ldots, x_{n}\right)$ is given by Eq. [27] for $k=2, \ldots, n-1$.

$$
\begin{aligned}
V\left[\delta_{k}(z-\zeta) / x_{1}, \ldots, x_{n}\right] & \\
& =\frac{\rho x_{k-1}\left(w_{k-1} / w_{k}\right)^{1 / 2}+w_{k} x_{k}+\rho x_{k+1}\left(w_{k+1} / w_{k}\right)^{1 / 2}}{\sum w_{i} x_{i}{ }^{2}}
\end{aligned}
$$


For $k=1$, the first term in the numerator is dropped, and for $k=n$ the last term is dropped. Using these estimates, with sample variances and the iterative estimates $z$ and $\bar{\rho}$ substituted one can examine the residuals, Eq. [28], as was done for the ratios directly.

$$
r_{k}=\frac{d_{k}}{v_{k}}=\frac{y_{k}-\hat{y}_{k}}{\left[V\left(y_{k}-\hat{y}_{k} / x_{1}, \ldots, x_{n}\right)\right]^{1 / 2}}
$$

We have not used this method much because of the large amount of computation involved. However, we have found that if we assume $\rho=0$, we obtain residual plots for the data shown in Fig. 6 that are similar to the residual plots in Fig. 7; the relative values of adjacent points differ somewhat, but the overall pattern is the same. We have also found that the serial correlation in the sequence $y_{i}-z x_{i}$ is much less than that in either $y_{i}$ or $x_{i}$ so that the assumption of only some small correlation between adjacent slices appears to be reasonable. The method deserves further exploration.

\section{DISCUSSION}

The problem of examining a sequence of ratios for deviation from a mean value is of considerable importance. Many types of labeling experiments with two isotopes are designed so that one expects uniform labeling in most fractions except in one or a few subsequences which deviate because the experimental treatment causes a change in labeling ratio in these samples. The problem is to identify subsequences in which the labeling ratio has been changed by the experimental treatment.

A number of methods for analyzing data from such experiments have been proposed. Yund et al. (4) calculated the standard deviations from counting statistics and for the ratio of isotope labeling. They wrote a program which provided a table of these summary statistics. However, their error calculations do not take into account the correlation between counts for the two isotopes in each gel. They do not consider tests for significant deviations from uniform labeling ratio. Weisberg (5) fitted the square roots of the isotope counts by a linear regression as in Eq. [29].

$$
y^{1 / 2}=a x^{1 / 2}+b
$$

He derived this on the assumption that the counts are Poissondistributed random variables. The parameters " $a$ " and " $b$ " are estimated by least squares and the deviations, $y_{i}{ }^{1 / 2}-\left(\hat{a} x_{i}{ }^{1 / 2}+\hat{b}\right)$, calculated; here $\hat{a}$ and $b$ are least squares estimates of " $a$ " and " $b$," respectively. He apparently also did not take into account the correlation between $x_{i}$ and $y_{i}$ within a slice and did not consider the effects of the serial correlations in the $x_{i}$ and $y_{i}$ on the regression estimates or on statistical tests of deviation. Mayol (6) used the excess counts $\Delta Y_{i}=y_{i}-x_{i} R_{m}$, where $R_{m}$ 
is the count weighted average ratio for the gel slices other than those in the peak that shows an apparent deviation from the general average. This is similar in some respects to the regression method but the weights used are not the correct weights for calculating the average ratio. Mayol does not consider the statistical estimation problems. More recently, Smith (7) has proposed that the fractional difference $F_{i}$, Eq. [30], be used to detect variation in the relative labelings by the two isotopes.

$$
F_{i}=\frac{x_{i}}{\sum_{j} x_{j}}-\frac{y_{i}}{\sum_{j} y_{j}}
$$

She also did not take into account the covariance between the two counts in one slice or the serial correlations between slices in calculating errors.

From our examination of data on gel slices it appears that the serial correlation is strong in the plot of DPM in each isotope but is either not significant or is small in plots of the ratio $y_{i} / x_{i}$ in controls or in subsequences of the experimental gels which show no large deviations from the mean ratio. The serial correlation becomes large when a deviation in ratio appears as in the example shown in Fig. 5.

The regression approach has many interesting features although the iterative computations are more involved than those required in the ratios directly. The apparently low serial correlation in the ratios and the fact that one can obtain high enough counts to obtain good sample estimates of the variances of the errors of measurement in the ratio argue for use of the ratio first in looking for significant deviations in labeling ratio. The one-way ANOVA as a rough test and the use of serial correlations in experimental designs that involve the proper controls, concentration, or other dependence of the experimental effect provide simple tests for significant deviations in labeling ratios. It is not really obvious that any refinements over these are needed for the experimental work involved.

\section{CONCLUSION}

The tests developed for analysis of deviations in isotope labeling ratios in experimental designs which use two isotopes have neglected important correlations in the data.

We derive estimates of the variance of the ratio of DPM of the two isotopes and conclude that the one-way ANOVA and serial correlations provide useful and simple ways of analyzing data on relative labeling. We find that a transformation to residuals of the labeling ratio provides a useful way of correlating the extent of change in labeling ratio with change in some experimental variable.

We have also developed the hasic theory for a regression model that appears to give results similar to those given by the ratio method but the computations are more demanding. 


\section{ACKNOWLEDGMENTS}

This work was supported in part by grants CA 06734 and GM 00110 from the NCI and $\mathrm{NIH}$, DHEW. We wish to thank the referees whose suggestions in the review of the first draft of this paper helped us to improve the paper considerably.

\section{REFERENCES}

1. Jung, C. Y. (1974) J. Biol. Chem. 249, 3568-3573.

2. Lin. S., and Spudich, J. A. (1974) J. Biol. Chem. 249, 5778-5783.

3. Fairbanks, G., Steck, T. L., and Wallach, D. F. H. (1971) Biochemistry 10, 2606-2617.

4. Yund, M. A., Yund, E. W., and Kafatos, F. D. (1971) Biochem. Biophys. Res. Commun. 43, 717-722.

5. Weisberg, S. (1974) Anal. Biochem. 61, 328-355.

6. Mayol, R. F. (1975) Mol. Cell. Endocrinol. 2, 133-146.

7. Smith, K. B. (1976) Anal. Biochem. 76, 16-31.

8. Bennett, C. A., and Franklin, N. L. (1961) Statistical Analysis in Chemistry and the Chemical Industry, New York.

9. Bush, E. T. (1964) Anal. Chem. 36, 1082-1089.

10. Kendall, M. G., and Stuart, A. (1961) The Advanced Theory of Statistics, Vol. 2, Sect. 20.34, Griffin, London.

11. Brownlee, K. A. (1965) Statistical Theory and Methodology in Science and Engineering, 2nd Ed., Sect. 3.21, Wiley, New York.

12. Ederer, F., and Mantel, N. (1974) Amer. J. Epidemiol. 11, 165-157.

13. Shanahan, M. F., and Jacquez, J. A. (1977) Membrane Biochem., in press. 